Pacific Journal of Mathematics

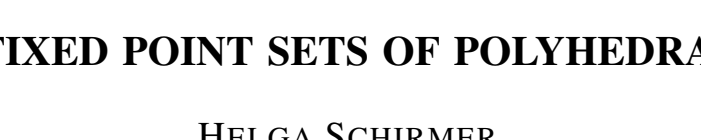




\section{FIXED POINT SETS OF POLYHEDRA}

\section{HELGA SCHIRMER}

It is shown that every closed nonempty subset of a polyhedron can be the fixed point set of a suitable self-map if the polyhedron satisfies a certain connectedness condition. Hence the same is true for all compact triangulable manifolds with or without boundary. The proof uses existing results on deformations of polyhedra with a minimum number of fixed points if the dimension of the polyhedron is at least two, and on self-maps of dendrites with given fixed point sets if the dimension of the polyhedron is one.

1. The ploblem. The following problem has been investigated by $\mathrm{H}$. Robbins, L. E. Ward, Jr., and the author in recent years: If $X$ is a topological space and $A$ a closed subset of $X$, when does there exist a continuous self-mapping of $X$ with $A$ as its fixed point set? Several cases are known where $A$ need not satisfy any restrictions apart from the obvious one that it must be nonempty if $X$ has the fixed point property.

L. E. Ward, Jr. [7] suggested the term "complete invariance property" if a space has the property that each of its nonempty closed subspaces can be the fixed point set of a self-map. Spaces known to have the complete invariance property include the $n$-cells [2], dendrites [4] and compact manifolds without boundary [6]. L. E. Ward, Jr. [7] extended this list with several other spaces, among them arcwise connected subspaces of locally smooth dendroids and a certain class of Peano continua. He also found an interesting example which shows that a tree need not have the complete invariance property, and hence that a generalization of existing results to nonmetric spaces presents difficulties.

It is the purpose of the present paper to show that all polyhedra which satisfy a certain connectedness condition have the complete invariance property. More precisely, they have to be of type $W$ (as defined in $\S 2$ ) if their dimension is greater than one (Theorem 3.1), and to be connected if their dimension is one (Theorem 3.2). It follows easily that all compact manifolds with or without boundary possess the complete invariance property (Corollary 3.3). The proof of Theorem 3.1 leans heavily on the construction of deformations of polyhedra with a minimum number of fixed points, which is now easily accessible in the recent book by R. F. Brown [1].

I wish to thank Professor L. E. Ward, Jr. for private communications of some of his results. 
2. Proximity maps. The proof of Theorem 3.1 below uses a proximity map of a polyhedron with exactly one given fixed point. The existence of such a map is established in this paragraph.

We denote by $|K|$ the polyhedron which is the realization of the finite simplicial complex $K$, by $|\sigma|$ an open simplex of $|K|$ and by $|\bar{\sigma}|$ its closure. The carrier $|\kappa(x)|$ of a point $x \in|K|$ is the unique simplex for which $x \in|\kappa(x)|$, the (open) star $\mid$ st $\sigma \mid$ of a simplex consists of all simplexes of $|K|$ which have $|\sigma|$ as a face. Let the open neighborhood $V(x)$ of a point $x \in|K|$ consist of all simplexes $|\sigma|$ of $|K|$ for which $|\bar{\kappa}(x)| \cap|\bar{\sigma}| \neq \varnothing$. Then $\mid$ st $\kappa(x) \mid \leqq V(x)$ for all $x \in|K|$. A map $f:|K| \rightarrow|K|$ is called a proximity map if $f(x) \in$ $V(x)$ for all $x \in|K|$. A proximity map is always a deformation (i.e., it is homotopic to the identity), as can be seen from the following lemma.

Lemma 2.1. Let $|K|$ be a polyhedron, I the unit interval, and

$$
N=\{(x, y) \in|K| \times|K||| \bar{\kappa}(x)|\cap| \bar{\kappa}(y) \mid \neq \varnothing\} .
$$

Then there exists a map $h: N \times I \rightarrow|K|$ such that

(1) $h(x, y, 0)=x$ and $h(x, y, 1)=y$,

(2) $h(x, x, t)=x$ for all $0 \leqq t \leqq 1$,

(3) $h(x, y, t) \neq x$ for all $x \neq y$ and $0<t \leqq 1$.

Proof. This is Lemma 1 of [1, p. 124].

A simplex $\sigma$ of $K$ is called maximal if it is not a (proper) face of any other simplex. The polyhedron $|K|$ is of type $W$ if every maximal simplex of $K$ is of dimension at least two, and if for every two maximal simplexes $\sigma, \sigma^{\prime}$ of $K$ there exist maximal simplexes $\sigma_{1}, \sigma_{2}, \cdots, \sigma_{r}$ such that $\sigma=\sigma_{1}, \sigma_{r}=\sigma^{\prime}$, and $\left|\bar{\sigma}_{i}\right| \cap\left|\bar{\sigma}_{i+1}\right|$ is of dimension at least one for $i=1,2, \cdots, r-1$. The Euler characteristic of $|K|$ is denoted by $\chi(|K|)$.

Lemma 2.2. Let $|K|$ be a polyhedron of type $W$. Then there exists a proximity map $f:|K| \rightarrow|K|$ which is fixed point free if $\chi(|K|)=0$, and which has exactly one fixed point a, contained in a maximal simplex, if $\chi(|K|) \neq 0$.

Proof. The map $f^{\prime}$ constructed in the proof of Theorem 1 in $[1$, p. 143] has the desired properties.

LEMmA 2.3. Let $\left|\sigma_{1}\right|,\left|\sigma_{2}\right|$ be two maximal simplexes of a polyhedron $|K|$ of type $W$. If $f_{1}:|K| \rightarrow|K|$ is a proximity map with a point $a_{1} \in\left|\sigma_{1}\right|$ as its only fixed point, then there exists a proximity 
map $f_{2}:|K| \rightarrow|K|$ with some point $a_{2} \in\left|\sigma_{2}\right|$ as its only fixed point.

Proof. As $f_{1}$ is a proximity map and hence a deformation, we see that the index $i\left(|K|, f_{1}, a_{1}\right)$ of $a_{1}$ under $f_{1}$ satisfies

$$
i\left(|K|, f_{1}, a_{1}\right)=L\left(f_{1}\right)=\chi(|K|) \neq 0,
$$

where $L\left(f_{1}\right)$ denotes the Lefschetz number of $f_{1}$. Hence the existence of $f_{2}$ follows from a repeated application of Lemma 3 of [1, p. 128]. (Note that the map $f^{\prime}$ constructed in this lemma satisfies $f^{\prime}(x)=f(x)$ if $x \notin U([a, b], \varepsilon)$.)

LEMmA 2.4. Let $|K|$ be a polyhedron of type $W$ and $\chi(|K|) \neq 0$. If $a$ is an arbitrary point of $|K|$, then there exists a proximity map $f:|K| \rightarrow|K|$ with a as its only fixed point.

Proof. Choose a maximal simplex $\sigma$ for which $a \in|\bar{\sigma}|$. Then it follows from Lemmas 2.2 and 2.3 that there exists a proximity map $f_{1}:|K| \rightarrow|K|$ with exactly one fixed point $b$, where $b \in|\sigma|$. Let $[a, b] \subset|\bar{\sigma}|$ be the line segment from $a$ to $b$, and $U([a, b], \eta)$ the $\eta$-neighborhood of $[a, b]$ in $|K|$ with closure $\bar{U}([a, b], \eta)$. Select $\eta>0$ so that $\bar{U}([a, b], \eta) \subset \mid$ st $\kappa(a) \mid$. For every point $x$ of the boundary $\dot{U}([a, b], \eta / 2)$ of $U([a, b], \eta / 2)$ there exists a $t^{*}=t^{*}(x)$ for which $0<t^{*} \leqq 1$ and

$$
h\left(x, f_{1}(x), t\right) \subset \mid \text { st } \kappa(a) \mid \text { if } \quad 0 \leqq t \leqq t^{*}(x) .
$$

As $\mid$ st $\kappa(a) \mid$ is open, the continuity of $h$ implies the existence of an $\varepsilon=\varepsilon(x)>0$ such that

$$
h\left(x^{\prime}, f_{1}\left(x^{\prime}\right), t\right) \subset \mid \text { st } \kappa(a) \mid
$$

for all $x^{\prime} \in \dot{U}([a, b], \eta / 2) \cap U(x, \varepsilon(x))$ and $0 \leqq t \leqq t^{*}(x)$. As $\dot{U}([a, b], \eta / 2)$ is compact, it follows that its cover

$$
\{U(x, \varepsilon(x)) \mid x \in \dot{U}([a, b], \eta / 2)\}
$$

has a finite subcover

$$
\left\{U\left(x_{i}, \varepsilon\left(x_{i}\right)\right) \mid x_{\imath} \in \dot{U}([a, b], \eta / 2) \quad \text { and } \quad i=1,2, \cdots, r\right\} .
$$

Let

$$
t_{0}=\min \left\{t^{*}\left(x_{1}\right), t^{*}\left(x_{2}\right), \cdots, t^{*}\left(x_{r}\right)\right\},
$$

then $0<t_{0} \leqq 1$. Hence $h\left(x, f_{1}(x), t_{0}\right) \subset \mid$ st $\kappa(a) \mid$ if $x \in \dot{U}([a, b], \eta / 2)$.

Now define a function $f:|K| \rightarrow|K|$ as follows: If $x \in|K| \mid \bar{U}([a, b], \eta)$, let $f(x)=f_{1}(x)$. If $x \in \bar{U}([a, b], \eta) \backslash\{a\}$, denote by $x(\eta / 2)$ resp. $x(\eta)$ the 
two unique points in which the ray $\overrightarrow{a x}$ from $a$ to $x$ intersects $\dot{U}([a, b], \eta / 2)$ resp. $\dot{U}([a, b], \eta)$. Let

$$
f(x)=h\left(x, f_{1}(x),\left(1-t_{0}\right) \lambda+t_{0}\right)
$$

if $x=(1-\lambda) x(\eta / 2)+\lambda x(\eta)$ and $0 \leqq \lambda \leqq 1$,

$$
f(x)=(1-\lambda) a+\lambda h\left(x(\eta / 2), f_{1}(x(\eta / 2)), t_{0}\right)
$$

if $x=(1-\lambda) \alpha+\lambda x(\eta / 2)$ and $0<\lambda \leqq 1$,

$$
f(a)=a .
$$

Then $f$ is well-defined and continuous, and hence the desired proximity map with fixed point $a$.

3. The complete invariance property of polyhedra. We now proceed to construct self-maps with arbitrarily given fixed point sets for polyhedra of type $W$ as well as for one-dimensional connected polyhedra.

THEOREM 3.1. Let $|K|$ be a polyhedron of type $W$, and $A$ be a closed subset of $|K|$ which is nonempty if $\chi(|K|) \neq 0$. Then $|K|$ admits a self-map with $A$ as its fixed point set.

Proof. We first assume that $\chi(|K|) \neq 0$. Then $A \neq \varnothing$, and we can select a point $a \in A$. Let $f:|K| \rightarrow|K|$ be a proximity map with $a$ as its only fixed point, which exists according to Lemma 2.4. For every $x \in|K|$, put $t(x)=d(x, A) / \delta$, where $\delta$ is the diameter of $|K|$ and $d(x, A)$ the distance of $x$ from $A$. Then $0 \leqq t(x) \leqq 1$, and $t(x)=0$ if and only if $x \in A$. Hence the map $g:|K| \rightarrow|K|$ defined by

$$
g(x)=h(x, f(x), t(x))
$$

is a self-map of $|K|$ with fixed point set $A$.

In the case $\chi(|K|)=0$, we know from Lemma 2.2 that there exists a fixed point free proximity map $f:|K| \rightarrow|K|$. If $A=\varnothing$, this is the desired self-map; if $A \neq \varnothing$, then a self-map $g$ with fixed point set $A$ can again be defined by $g(x)=h(x, f(x), t(x))$.

THEOREM 3.2. Let $|K|$ be a one-dimensional connected polyhedron, and $A$ be a closed subset of $|K|$ which is nonempty if $\chi(|K|) \neq 1$. Then $|K|$ admits a self-map with $A$ as its fixed point set.

Proof. (i ) If $\chi(|K|)=1$, then $|K|$ is acyclic and hence a metric tree. In this case Theorem 3.2 follows from [4, Theorem 3.1]. 
(ii) Now assume $\chi(|K|) \neq 1$ and $A=\varnothing$. Select a maximal subtree $|T|$ of $|K|$, so that

$$
|K|=|T| \cup\left|\sigma_{1}\right| \cup\left|\sigma_{2}\right| \cup \cdots \cup\left|\sigma_{r}\right|,
$$

where $\left|\sigma_{i}\right|$ are one-simplexes and $r \geqq 1$. Take a point $x_{0}$ in the interior of $\left|\bar{\sigma}_{1}\right|=[v, w]$, and construct a closed and oriented arc based at $x_{0}$ which starts with the segment $\left[x_{0}, w\right]$ in $\left|\bar{\sigma}_{1}\right|$, then connects the points $w$ and $v$ in $|T|$, and ends with the segment $\left[v, x_{0}\right]$ in $\left|\bar{\sigma}_{1}\right|$. Put $f\left(K|/| \sigma_{1} \mid\right)=x_{0}$, and map $\left|\bar{\sigma}_{1}\right|$ onto the chosen arc in a suitable manner to obtain a fixed point free self-map of $|K|$.

(iii) Finally assume $\chi(|K|) \neq 1$ and $A \neq \varnothing$. This time, select a maximal subtree $|T|$ of $|K|$ such that $|T| \cap A \neq \varnothing$. Again it follows from [4, Theorem 3.1] that there exists a map $f:|T| \rightarrow|T|$ with fixed point set $|T| \cap A$. It remains to extend $f$ over each $\left|\sigma_{i}\right|$ of

$$
|K|=|T| \cup\left|\sigma_{1}\right| \cup\left|\sigma_{2}\right| \cup \cdots \cup\left|\sigma_{r}\right|
$$

with fixed point set $\left|\sigma_{i}\right| \cap A$.

If $\left|\sigma_{i}\right| \cap A=\varnothing$, choose any arc in $|T|$ from $f\left(v_{i}\right)$ to $f\left(w_{i}\right)$, where $v_{i}$ and $w_{i}$ are the end-points of $\left|\bar{\sigma}_{i}\right|$, and map $\left|\bar{\sigma}_{i}\right|$ onto it. If $\left|\sigma_{i}\right| \cap$ $A \neq \varnothing$, let $a_{i}$ resp. $b_{i}$ be the points of $\left|\sigma_{i}\right| \cap A$ closest to $v_{i}$ resp. $w_{i}$. (The points $a_{i}$ and $b_{i}$ need not be distinct.) Select an arc in $|T| \cup\left|\sigma_{i}\right|$ from $f\left(v_{i}\right)$ via $v_{i}$ to $a_{i}$ and define $f$ on the segment $\left[v_{i}, a_{i}\right]$ as a homeomorphism onto this arc which is fixed point free on $\left(v_{i}, a_{i}\right)$. In the same way, map $\left[b_{i}, w_{i}\right]$ onto an arc in $|T| \cup\left|\sigma_{i}\right|$ from $b_{i}$ via $w_{i}$ to $f\left(w_{i}\right)$. Lastly map the segment $\left[a_{\imath}, b_{\imath}\right]$ onto itself with fixed point set $\left[a_{i}, b_{i}\right] \cap A$, which is obviously possible (see e.g. [3, Theorem 7]).

In this way we can extend $f$ over each $\left|\sigma_{i}\right|$, and therefore over $|K|$, to a self-map of $|K|$ with fixed point set $A$.

A connected polyhedron $|K|$ is a compact and triangulable manifold of dimension $n$ if every point $x \in|K|$ has a neighborhood $U(x)$ such that $U(x)$ is homeomorphic to either Euclidean $n$-space $R^{n}$ or to $H^{n}=\left\{\left(x_{1}, x_{2}, \cdots, x_{n}\right) \in R^{n} \mid x_{n} \geqq 0\right\}$. If the set of points with a neighborhood homeomorphic to $H^{n}$ is nonempty, then $|K|$ is a manifold with boundary, otherwise a manifold without boundary. It follows from [6, Theorem 4] that a manifold without boundary has the complete invariance property. We can use Theorems 3.1 and 3.2 to extend this result to manifolds with boundary.

COROLlARY 3.3. A triangulable manifold with or without boundary has the complete invariance property.

Proof. If the dimension of the manifold $|K|$ is at least two, then $|K|$ is a polyhedron of type $W$ (compare [1, p. 145]), and hence 
Corollary 3.3 follows from Theorem 3.1. If $|K|$ is of dimension one, then the result is a consequence of Theorem 3.2.

The result proved in [6, Theorem 4] for manifolds without boundary is actually stronger: It shows that any closed subset can be the fixed point set of a surjective self-map. This is certainly not true for one-dimensional manifolds with boundary, as an end-point of an interval cannot be the fixed point set of a surjection. It is not yet known which conditions a closed subset of a manifold with boundary of dimension greater than one, let alone of a connected polyhedron, has to satisfy so that it can be the fixed point set of a surjective self-map. It might be of interest to note in this context that necessary and sufficient conditions for fixed point sets of surjections of dendrites have been determined by L. E. Ward, Jr. [7, Theorem 10]. We further know some properties of fixed point sets of homeomorphisms for the special cases of $n$-balls [3], compact surfaces [6], spheres [6], and dendrites [4, 5], but not for more general spaces.

It seems possible that the condition in Theorem 3.1 that $|K|$ must be of type $W$ can be relaxed, and that Corollary 3.3 can be proved for manifolds with boundary which are not necessarily triangulable, as was done in [6, Theorem 4] for manifolds without boundary.

\section{REFERENCES}

1. R. F. Brown, The Lefschetz Fixed Point Theorem, Scott, Foresman and Co., Glenview, Ill., 1971.

2. H. Robbins, Some complements to Brouwer's fixed point theorem, Israel J. Math., 5 (1967), 225-226.

3. H. Schirmer, On fixed point sets of homeomorphisms of the n-ball, Israel J. Math., 7 (1969), 46-50.

4. - Properties of fixed point sets on dendrites, Pacific J. Math., 36 (1971), $795-810$.

5. — Fixed point sets of homeomorphisms on dendrites, Fund. Math., 75 (1972), 117-122.

6. - Fixed point sets of homeomorphisms of compact surfaces, Israel J. Math., 10 (1971), 373-378.

7. L. E. Ward, Jr., Fixed point sets, Pacific J. Math., 47 (1973), 553-565.

Received December 14, 1972. This research was partially supported by the National Research Council of Canada (Grant A 7579).

Carleton University, Ottawa, Canada 


\section{PACIFIC JOURNAL OF MATHEMATICS}

EDITORS

RICHARD ARENS (Managing Editor)

University of California

Los Angeles, California 90024

R. A. Beaumont

University of Washington

Seattle, Washington 98105

J. DugundJI

Department of Mathematics

University of Southern California

Los Angeles, California 90007

D. Gilbarg and J. Milgram

Stanford University

Stanford, California 94305

\section{ASSOCIATE EDITORS}

E. F. BECKENBACH

B. H. NeumanN

F. WOLF

K. YOSHIDA

\section{SUPPORTING INSTITUTIONS}

UNIVERSITY OF BRITISH COLUMBIA CALIFORNIA INSTITUTE OF TECHNOLOGY

UNIVERSITY OF CALIFORNIA

MONTANA STATE UNIVERSITY

UNIVERSITY OF NEVADA

NEW MEXICO STATE UNIVERSITY

OREGON STATE UNIVERSITY

UNIVERSITY OF OREGON

OSAKA UNIVERSITY

\author{
UNIVERSITY OF SOUTHERN CALIFORNIA \\ STANFORD UNIVERSITY \\ UNIVERSITY OF TOKYO \\ UNIVERSITY OF UTAH \\ WASHINGTON STATE UNIVERSITY \\ UNIVERSITY OF WASHINGTON \\ $\stackrel{*}{*} \stackrel{*}{*}{ }^{*}{ }^{*}{ }^{2}$ AMERICAN MATHEMATICAL SOCIETY \\ NAVAL WEAPONS CENTER
}




\section{Pacific Journal of Mathematics}

\section{Vol. 52, No. $1 \quad$ January, 1974}

David R. Adams, On the exceptional sets for spaces of potentials ............ 1

Philip Bacon, Axioms for the Čech cohomology of paracompacta ............ 7

Selwyn Ross Caradus, Perturbation theory for generalized Fredholm operators ..... 11

Kuang-Ho Chen, Phragmén-Lindelöf type theorems for a system of nonhomogeneous equations ............................ 17

Frederick Knowles Dashiell, Jr., Isomorphism problems for the Baire classes .......

M. G. Deshpande and V. K. Deshpande, Rings whose proper homomorphic images are right subdirectly irreducible . . . . . . . . . . . . . . . . . . . . . . . . .

Mary Rodriguez Embry, Self adjoint strictly cyclic operator algebras .............

Paul Erdős, On the distribution of numbers of the form $\sigma(n) / n$ and on some related

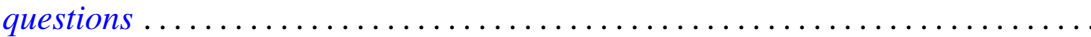

Richard Joseph Fleming and James E. Jamison, Hermitian and adjoint abelian

operators on certain Banach spaces ............................

Stanley P. Gudder and L. Haskins, The center of a poset .................. 85

Richard Howard Herman, Automorphism groups of operator algebras . . . ........

Worthen N. Hunsacker and Somashekhar Amrith Naimpally, Local compactness of families of continuous point-compact relations ....................

Donald Gordon James, On the normal subgroups of integral orthogonal groups ....

Eugene Carlyle Johnsen and Thomas Frederick Storer, Combinatorial structures in

loops. II. Commutative inverse property cyclic neofields of prime-power

order.

Ka-Sing Lau, Extreme operators on Choquet simplexes . . . . . . . . . . . . . . 129

Philip A. Leonard and Kenneth S. Williams, The septic character of 2, 3, 5 and $7 \ldots 143$

Dennis McGavran and Jingyal Pak, On the Nielsen number of a fiber map ........ 149

Stuart Edward Mills, Normed Köthe spaces as intermediate spaces of $L_{1}$ and

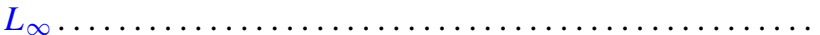

Philip Olin, Free products and elementary equivalence. .

Louis Jackson Ratliff, Jr., Locally quasi-unmixed Noetherian rings and ideals of the principal class.

Seiya Sasao, Homotopy types of spherical fibre spaces over spheres ...

Helga Schirmer, Fixed point sets of polyhedra ...

Kevin James Sharpe, Compatible topologies and continuous irreducible

representations.

Frank Siwiec, On defining a space by a weak base . . . . . . . . . . . . . . . 233

James McLean Sloss, Global reflection for a class of simple closed curves ....... 247

M. V. Subba Rao, On two congruences for primality . .

Raymond D. Terry, Oscillatory properties of a delay differential equation of even

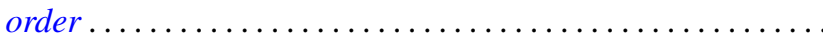

Joseph Dinneen Ward, Chebyshev centers in spaces of continuous functions . .

Robert Breckenridge Warfield, Jr., The uniqueness of elongations of Abelian

groups...

V. M. Warfield, Existence and adjoint theorems for linear stochastic differential

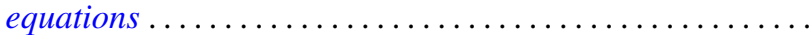

